TRANSACTIONS OF THE

AMERICAN MATHEMATICAL SOCIETY

Volume 355, Number 8, Pages 3241-3252

S 0002-9947(03)03308-7

Article electronically published on April 16, 2003

\title{
HAUSDORFF DIMENSION AND ASYMPTOTIC CYCLES
}

\author{
MARK POLLICOTT
}

\begin{abstract}
We carry out a multifractal analysis for the asymptotic cycles for compact Riemann surfaces of genus $g \geq 2$. This describes the set of unit tangent vectors for which the associated orbit has a given asymptotic cycle in homology.
\end{abstract}

\section{INTRODUCTION}

For many years Hausdorff dimension has played an important role in understanding some subtle features in both geometry and dynamical systems. In this note we use it as a tool in studying geodesic flows and the homology of surfaces. Let $V$ be a compact oriented Riemann surface with genus $g \geq 2$ and consider the geodesic flow $\phi_{t}: S_{1} V \rightarrow S_{1} V$ on the unit tangent bundle. We can associate to each $\phi$ invariant probability measure $\mu$ a Schwartzman asymptotic cycle $\Lambda_{\mu} \in H^{1}(V, \mathbb{R})^{*}$. By Poincaré duality we can identify $H_{1}(V, \mathbb{R})=H^{1}(V, \mathbb{R})^{*}$ and we can trivially identify $H_{1}(V, \mathbb{R})=\mathbb{R}^{2 g}$.

There is a particularly simple geometric way to understand $\Lambda_{\mu}$ (cf. $[\mathrm{Su}]$ ). Given $v \in S_{1} M$ and large $T$ we can close up the orbit segment $\phi_{[0, T]} v$ by an arc of uniformly bounded length to get a closed curve $\gamma_{v, T}$, say. Then for almost every $v$ (with respect to $\mu$ ) we have that $\left[\gamma_{v, T}\right] / T \rightarrow \Lambda_{\mu}$ as $T \rightarrow \infty$. The range of values $\mathcal{B} \subset H_{1}(V, \mathbb{R})$ that can occur for different invariant measures is the "unit ball in the stable norm". We shall prove the following result.

Theorem 1. For $\underline{\alpha} \in \operatorname{int}(\mathcal{B})$ in the interior of $\mathcal{B}$, the set of unit tangent vectors $X_{\underline{\alpha}} \subset S_{1} V$ for which the limit exists and equals $\alpha$ is dense and uncountable. Moreover, the map $\operatorname{int}(\mathcal{B}) \ni \underline{\alpha} \rightarrow \operatorname{dim}_{H}\left(X_{\underline{\alpha}}\right)$ is analytic.

Our approach uses a multivariable multifractal result on interval maps (Lemma 5 ), for which we give a simple proof based on the elegant argument in [ $\mathrm{PW}]$. Previously, Pesin and Sadovskaya studied multifractal analysis for Anosov flows [PS], but the exact type of multivariable results we require are not covered there. On the other hand, Barreira, Saussol and Schmeling have developed a general theory of multivariable multifractal analysis [BSS], but their results are formulated from the point of view of entropies, rather than Hausdorff dimension (cf. [Je2]).

Received by the editors October 25, 2002.

2000 Mathematics Subject Classification. Primary 28A78, 37D35; Secondary 37D40, 55N10.

I am very grateful to Howie Weiss and Luis Barreira for very useful conversations on multifractal analysis.

(C)2003 American Mathematical Society 


\section{Asymptotic CyCles AND the SET $\mathcal{B}$}

We begin with some general background and useful results on asymptotic cycles. The following definition was introduced by Schwartzman in 1957 Sc].

Definition. To each $\phi$-invariant measure $\mu$ we associate a linear functional $\Lambda_{\mu} \in$ $H^{1}(V, \mathbb{R})^{*}\left(=H_{1}(V, \mathbb{R})\right)$ by

$$
\Lambda_{\mu}(\omega)=\int \omega(v) d \mu(v)
$$

where $\omega$ is a closed 1-form representing an element $[\omega] \in H^{1}(V, \mathbb{R})$ in the de Rham cohomology. We call $\Lambda_{\mu}$ the asymptotic cycle for $\mu$.

The following result is well known.

Lemma 1 [Sc]. If $\lambda$ is the Liouville measure, then $\Lambda_{\lambda}=0$.

Proof. Since the proof is simple, we include it for completeness. Geodesic flows have a natural involution $i: S_{1} V \rightarrow S_{1} V$ so that the image $i(v)$ of a unit tangent vector $v$ has the same base point, but points in the opposite direction. Since $i_{*} \lambda=\lambda$, it is easy to see that $\Lambda_{\lambda}(\omega)=\int \omega(v) d \lambda(v)=0$ for all closed 1-forms $\omega$.

For other simple examples of Anosov Hamiltonian flows the Liouville measure will still be preserved, but the corresponding asymptotic cycle may well be nonzero.

Definition. The Federer stable norm $\|\cdot\|$ on $H_{1}(V, \mathbb{R})$ is defined by

$$
\|v\|=\inf \left\{\sum_{i}\left|r_{i}\right| \cdot \operatorname{length}\left(\gamma_{i}\right): v=\sum_{i} r_{i} \gamma_{i} \text { with } r_{i} \in \mathbb{R}, \gamma_{i}=\text { closed curve }\right\} \text {. }
$$

Let $\mathcal{B} \subset H_{1}(V, \mathbb{R})$ denote the closed unit ball with respect to the stable norm.

A well-known classical conjecture (related to the Hopf conjecture cf. [BI]) states that the stable norm for a torus is Euclidean if and only if the metric is flat. Bangert showed that the stable norm of a 2-torus is differentiable in a rational direction if and only if the corresponding minimizing geodesics foliate the torus $[\mathrm{Ba}]$. The set $\mathcal{B}$ was studied for surfaces of higher genus by McShane and Rivin [MR] and Massart [Ma1], Ma2]. McShane and Rivin showed that the stable norm for a oncepunctured torus is never differentiable in a rational direction, and Massart extended this to compact oriented Riemann surfaces $V$ with genus $g \geq 2$.

Lemma 2 Ma2]. The set $\mathcal{B}$ is closed and convex. However, $\mathcal{B}$ is not strictly convex and the boundary is not differentiable in rational directions.

Let us consider a more dynamical viewpoint. For each (directed) closed geodesic $\gamma$ we can associate its real homology class $[\gamma] \in H_{1}(V, \mathbb{R})$ and its length $l(\gamma)$. Two equivalent presentations of $\mathcal{B} \subset H_{1}(V, \mathbb{R})$ are (cf. Ma1]

$$
\begin{aligned}
\mathcal{B} & =\left\{\Lambda_{\mu}: \mu=\phi-\text { invariant probability }\right\} \\
& =\left\{\frac{[\gamma]}{l(\gamma)}: \gamma=\text { is a closed geodesic }\right\}
\end{aligned}
$$

Observe that by fixing a suitable basis of (harmonic) 1 -forms $\omega_{1}, \ldots, \omega_{n}$ for $H^{1}(V, \mathbb{R})$ we can write $\Lambda_{\mu}=\alpha_{1} \omega_{1}+\ldots+\alpha_{2 g} \omega_{2 g}$ and therefore represent $\Lambda_{\mu}$ in coordinates 
as $\underline{\alpha}=\left(\alpha_{1}, \ldots, \alpha_{2 g}\right)$. We can now define functions $f_{i}: S_{1} V \rightarrow \mathbb{R}(i=1, \ldots, 2 g)$ on the unit tangent bundle by $f_{i}(v)=\omega_{i}(v)$ and rewrite

$$
X_{\underline{\alpha}}=\left\{v \in S_{1} V: \lim _{T \rightarrow \infty} \frac{1}{T} \int_{0}^{T} f_{j}\left(\phi_{t} v\right) d t=\alpha_{j}, \text { for } j=1, \ldots, 2 g\right\} .
$$

We briefly recall the definition of Hausdorff dimension. Let $(Y, d)$ be a metric space. Given $\epsilon, \delta>0$ we can define

$$
H_{\delta}^{s}(Y)=\inf \left\{\sum_{U \in \mathcal{U}} \operatorname{diam}(U)^{s}: \mathcal{U}=\delta \text {-cover for } Y\right\},
$$

where the summation is over all covers $\mathcal{U}$ consisting of open sets of diameter at most $\delta$. We can then let $H^{s}(Y)=\lim _{\delta \rightarrow 0} H_{\delta}^{s}(Y)$ and define the Hausdorff dimension of $Y$ by $\operatorname{dim}_{H}(Y)=\inf \left\{\delta>0: H^{\delta}(Y)=0\right\}$. We can write $S_{1} V$ as a disjoint union

$$
S_{1} V=X_{0} \cup\left(\bigcup_{\underline{\alpha} \in H_{1}(V, \mathbb{R})-\{0\}} X_{\underline{\alpha}}\right) \cup X_{\infty}
$$

where

(a) $X_{0}=\left\{v \in X: \lim _{T \rightarrow+\infty}\left[\gamma_{v, T}\right] / T=0\right\}$ has full Liouville measure;

(b) $X_{\underline{\alpha}}=\left\{v \in X: \lim _{T \rightarrow+\infty}\left[\gamma_{v, T}\right] / T=\underline{\alpha}\right\}$ has zero Liouville measure for $\underline{\alpha} \neq 0$

(c) $X_{\infty}$ is the set of points for which $\left[\gamma_{v, T}\right] / T$ does not converge.

We recall that a $\phi$-invariant probability measure $\mu$ is a Gibbs measure (for a Hölder continuous function $F: S_{1} V \rightarrow \mathbb{R}$, say) if

$$
h(\phi, \mu)+\int F d \mu \geq h(\phi, \nu)+\int F d \nu
$$

for all $\phi$-invariant probability measures $\nu$. The following more precise result subsumes Theorem 1.

\section{Proposition 1.}

(1) The Hausdorff dimension $\operatorname{dim}_{H}\left(X_{\underline{\alpha}}\right)$ satisfies $\operatorname{dim}_{H}\left(X_{\underline{\alpha}}\right) \leq 3$, with equality if and only if $\underline{\alpha}=0$;

(2) The Hausdorff dimension $\operatorname{dim}_{H}\left(X_{\underline{\alpha}}\right)$ depends real analytically on $\underline{\alpha} \in$ $\operatorname{int}(\mathcal{B})$;

(3) For $\underline{\alpha} \in \operatorname{int}(\mathcal{B})$, the set $X_{\underline{\alpha}} \subset S_{1} V$ is dense and uncountable and carries a Gibbs measure.

Remark. By contrast, if $\underline{\alpha}$ is a point in the boundary of $\mathcal{B}$, then $\Lambda_{\underline{\alpha}}$ may consist of a single orbit (corresponding to a simple closed geodesic) [Ma1, [Ma2].

To prove Proposition 1 it is convenient to reduce the problem to one for interval maps. In particular, the following standard result helps to relate the flow to a simple one-dimensional system.

Lemma 3. There exists an expanding $C^{\omega}$ Markov interval map $T: I \rightarrow I$ and locally constant functions $\psi_{i}: I \rightarrow \mathbb{R}(i=1, \ldots, 2 g)$ such that:

(1) the prime closed $\phi$-orbits $\gamma$ correspond to prime periodic $T: I \rightarrow I$ orbits $T^{n} x=x$ (with at most a finite number of exceptions);

(2) the least period of $\gamma$ is given by $l(\gamma)=\log \left|\left(T^{n}\right)^{\prime}(x)\right|$; 
(3) the homology class of $\gamma$ is given by $[\gamma]=\left(\psi_{1}^{n}(x), \ldots, \psi_{2 g}^{n}(x)\right)$, where we write $\psi_{i}^{n}(x)=\psi(x)+\psi_{i}(T x)+\cdots+\psi_{i}\left(T^{n-1} x\right)$, for $1 \leq i \leq 2 g$ and $n \geq 1$.

Proof. The construction is fairly standard; so we shall only give an outline. We begin by choosing (two-dimensional) Markov Poincaré sections $T_{1}, \ldots, T_{k}$ to the geodesic flow $\phi_{t}: S_{1} V \rightarrow S_{1} V$ [Ra], Bw1]. Moreover, we can assume that the sections are foliated by stable manifolds. In particular, we can identify the sections along the stable manifolds so that each section $T_{i}$ quotients to an interval $I_{i}$, say. By virtue of the Markov property of the sections, the Poincaré (first return) map on $\bigcup_{i=1}^{n} T_{i}$ induces a Markov interval map $T: I \rightarrow I$, where $I=\bigcup_{i=1}^{n} I_{i}$. Since the foliation of $S_{1} V$ by stable manifolds is analytic, we can deduce that $T: I \rightarrow I$ is $C^{\omega}$. There is a one-to-one correspondence between closed $\phi$-orbits $\gamma$, periodic orbits for the Poincaré map and $T$-periodic points, except possibly for the (at most) finite number of closed $\phi$-orbits that pass through the boundary of a section.

For part(2), it is an easy observation that since the surface has constant curvature $\kappa=-1$, say, the length $l(\gamma)$ is equal to the expansion coefficient (or Lyapunov exponent) in the unstable direction around the orbit. If $\gamma$ corresponds to the periodic orbit $T^{n} x=x$, then the associated expansion coefficient around the $T$ orbit is precisely $\log \left|\left(T^{n}\right)^{\prime}(x)\right|$.

For part (3), we follow a construction of Franks $[\mathrm{Fr}]$. We can choose a fixed reference point $x_{0} \in V$ and continuous paths $\rho_{i}:[0,1] \rightarrow V(i=1, \ldots, k)$ such that $\rho_{i}(0)=x_{0}$ and $\rho_{i}(1)$ is contained in the image $\pi\left(T_{i}\right)$ under the canonical projection $\pi: S_{1} V \rightarrow V$. Assuming that $T^{-1}\left(\operatorname{int}\left(I_{i}\right)\right) \cap \operatorname{int}\left(I_{j}\right) \neq \emptyset$, we can associate a closed curve $c_{i j}$ consisting of the composition of $\rho_{i}$; a curve approximating the geodesic arc from $\pi\left(T_{i}\right)$ to $\pi\left(T_{j}\right)$; and $\rho_{j}^{-1}$. We let $\left[c_{i j}\right] \in H_{1}(V, \mathbb{R})=\mathbb{R}^{2 g}$ be the associated element in homology. We can then define $\psi_{i}: I \rightarrow \mathbb{R}$ by the coordinate functions, i.e., $\left(\psi_{1}(x), \ldots, \psi_{2 g}(x)\right)=\left[c_{i j}\right]$ when $x \in \operatorname{int}\left(I_{i}\right)$ and $T x \in \operatorname{int}\left(I_{j}\right)$. In particular, we see that if $T^{j} x \in I_{i_{j}}$, for $j=0, \ldots, n-1$, then

$$
\begin{aligned}
{[\gamma] } & =\left[c_{i_{0} i_{1}}\right] \circ \ldots \circ\left[c_{i_{n-2} i_{n-1}}\right] \circ\left[c_{i_{n-1} i_{0}}\right] \\
& =\left(\psi_{1}^{n}(x), \ldots, \psi_{2 g}^{n}(x)\right) .
\end{aligned}
$$

Moreover, the functions $\psi_{i}$ are constant on $T^{-1}\left(\operatorname{int}\left(I_{j}\right)\right) \cap \operatorname{int}\left(I_{i}\right)$.

To prove Proposition 1, we shall want to apply the following general result on Markov interval maps.

Lemma 4. Let $T: I \rightarrow I$ be an expanding $C^{1+\beta}$ Markov interval map, for some $0<\beta \leq 1$, and let $\psi_{i}: I \rightarrow \mathbb{R}$ (for $\left.i=1, \ldots, N\right)$ be $C^{\beta}$ functions. Let $\underline{\alpha}=$ $\left(\alpha_{1}, \ldots, \alpha_{N}\right) \in \mathbb{R}^{N}$. Let

$$
Y_{\underline{\alpha}}(T, I)=\left\{\omega \in I: \lim _{n \rightarrow+\infty} \frac{\psi_{j}^{n}(\omega)}{\log \left|\left(T^{n}\right)^{\prime}(\omega)\right|}=\alpha_{j}, \text { for } j=1, \ldots, N\right\} .
$$

Let $\mathcal{B} \subset \mathbb{R}^{N}$ be the range of $\left(\frac{\int \psi_{1} d m}{\int \log \left|T^{\prime}\right| d m}, \cdots, \frac{\int \psi_{N} d m}{\int \log \left|T^{\prime}\right| d m}\right)$, where $m$ ranges over all $T$-invariant probability measures. For $\underline{\alpha} \in \operatorname{int}(\mathcal{B})$ we have that:

(1) The Hausdorff dimension $\operatorname{dim}_{H}\left(Y_{\underline{\alpha}}(T, I)\right)$ satisfies $\operatorname{dim}_{H}\left(Y_{\underline{\alpha}}(T, I)\right) \leq 1$, with equality if and only if $\underline{\alpha}=0$;

(2) The Hausdorff dimension $\operatorname{dim}_{H}\left(Y_{\underline{\alpha}}(T, I)\right)$ depends real analytically on $\underline{\alpha}$ on the interior of $\mathcal{B}$; 
(3) For $\underline{\alpha} \in \operatorname{int}(\mathcal{B})$, the set $Y_{\underline{\alpha}}(T, I) \subset I$ is dense, uncountable and carries a Gibbs measure.

Lemma 4 can be deduced as a special case of Theorems 8 and 13 in BSS (and observation 2 after Theorem 8). However, we can present a self-contained independent proof of this lemma in this paper.

Lemma 5. We can identify $\operatorname{dim}_{H}\left(X_{\underline{\alpha}}\right)=\operatorname{dim}_{H}\left(Y_{\underline{\alpha}}(T, I)\right)+2$.

Proof. It is immediate from the definitions that $X_{\alpha}$ is $\phi$-invariant. In particular, we can write that $\operatorname{dim}_{H}\left(X_{\underline{\alpha}}\right)=\operatorname{dim}_{H}\left(X_{\underline{\alpha}} \cap\left(\bigcup_{i=1}^{k} T_{i}\right)\right)+1=\max _{1 \leq i \leq k} \operatorname{dim}_{H}\left(X_{\underline{\alpha}} \cap\right.$ $\left.T_{i}\right)+1$. Moreover, if $v \in X_{\underline{\alpha}} \cap T_{i}$, for some $1 \leq i \leq k$, then all points lie on the same piece of stable manifold in $T_{i}$ as $v$. Since the foliation of each section $T_{i}$ is Lipschitz (even $C^{\omega}$ ), we deduce that $\operatorname{dim}_{H}\left(X_{\underline{\alpha}} \cap T_{i}\right)=\operatorname{dim}_{H}\left(Y_{\underline{\alpha}}(T, I)\right)+1$. This completes the proof.

Proposition 1 now follows from Lemmas 4 and 5. A slight modification of this analysis gives the following stronger result.

Proposition 2. Given $\underline{\alpha}, \underline{\beta} \in H_{1}(V, \mathbb{R})$, let $X_{\alpha, \beta} \subset S_{V}$ be the set of unit tangent vectors $v$ such that $\lim _{T \rightarrow \infty}\left[\gamma_{v, T}\right] / T=\underline{\alpha}$ and $\lim _{T \rightarrow \infty}\left[\gamma_{v,-T}\right] / T=\underline{\beta}$ (by which we denote the asymptotic cycle flowing backwards in time).

(1) The Hausdorff dimension $\operatorname{dim}_{H}\left(X_{\underline{\alpha}, \underline{\beta}}\right)$ satisfies $\operatorname{dim}_{H}\left(X_{\underline{\alpha}, \underline{\beta}}\right) \leq 3$, with equality if and only if $\underline{\alpha}=\beta=0$;

(2) The Hausdorff dimension $\overline{\operatorname{dim}}_{H}\left(X_{\underline{\alpha}}, \underline{\beta}\right)$ depends real analytically on $\underline{\alpha}, \underline{\beta} \in$ $\operatorname{int}(\mathcal{B})$;

(3) For $\underline{\alpha}, \underline{\beta} \in \operatorname{int}(\mathcal{B})$, the set $X_{\underline{\alpha}, \underline{\beta}} \subset S_{1} V$ is dense and uncountable and carries a Gibbs measure.

Proof. If we had chosen in the proof of Lemma 3 to change the direction of the flow, i.e., replace the flow $\phi_{t}$ by the flow $\phi_{-t}$, then this would have had the effect of reversing the direction of the Poincaré map and interchanging the stable and unstable manifolds. Following the same steps in the construction as before we would arrive at another $C^{\omega}$ expanding Markov map $S: J \rightarrow J$, say. By a similar reasoning to that above we can deduce that $X_{\underline{\alpha}, \underline{\beta}} \cap T_{i}$ is locally diffeomorphic to $Y_{\underline{\alpha}}(T, I) \times Y_{\underline{\beta}}(S, J)$, for each $1 \leq i \leq k$. Moreover, we can write $\operatorname{dim}_{H}\left(X_{\underline{\alpha}, \underline{\beta}}\right)=$ $\operatorname{dim}_{H}\left(Y_{\underline{\alpha}}(T, \bar{I})\right)+\operatorname{dim}_{H}\left(Y_{\underline{\beta}}(S, J)\right)+1$. The result then follows from Lemma 4 .

\section{Subshifts and Gibbs measures}

We now need to do some preliminary work in order to prove Lemma 4 . The expanding Markov interval map $T: I \rightarrow I$ can be modelled by a one-sided subshift of finite type. More precisely, we define the $k \times k$ matrix $A$ by $A(i, j)=1$ if $T^{-1} \operatorname{int}\left(T_{j}\right) \cap \operatorname{int}\left(T_{i}\right) \neq \emptyset$, and 0 otherwise. We can define

$$
\Sigma_{A}^{+}=\left\{x \in \prod_{n=0}^{\infty}\{1, \ldots, k\}: A\left(x_{n}, x_{n+1}\right)=1, n \geq 0\right\}
$$

and a metric $d(x, y)=\sum_{n=0}^{\infty} 2^{-n}\left(1-\delta\left(x_{n}, y_{n}\right)\right)$. We define a local homeomorphism $\sigma: \Sigma_{A}^{+} \rightarrow \Sigma_{A}^{+}$by $(\sigma x)_{n}=x_{n+1}$. The Hölder map $\pi: \Sigma_{A}^{+} \rightarrow I$ defined by $\pi(x)=$ $\bigcap_{n=0}^{\infty} T^{-n} I_{x_{n}}$ is a semi-conjugacy i.e., $\pi \sigma=T \pi$. We define Hölder functions $\widehat{\psi}_{j}$ : $\Sigma_{A}^{+} \rightarrow \mathbb{R}$, for $j=1 \ldots, 2 g$ by $\widehat{\psi}_{j}=\psi_{j} \circ \pi$. 
To proceed we need to impose the following hypothesis on the functions.

Hypothesis. There are no solutions $b_{1}, \ldots, b_{2 g} \in \mathbb{R}$ and $u \in C\left(\Sigma_{A}^{+}, \mathbb{R}\right)$ to

$$
\log \left|T^{\prime}\right| \circ \pi+\sum_{j=1}^{2 g} b_{j} \widehat{\psi}_{j}+u \circ \sigma-u=0 .
$$

It is easy to check that this hypothesis holds in our application by considering closed orbits. In particular, the above identity would imply that the lengths of closed geodesics lie in the semi-group $b_{1} \mathbb{Z}^{+}+\ldots+b_{2 g} \mathbb{Z}^{+}$, giving an obvious contradiction.

We define the pressure map $P: C^{\gamma}\left(\Sigma_{A}^{+}, \mathbb{R}\right) \rightarrow \mathbb{R}$ by

$$
P(g)=\limsup _{n \rightarrow+\infty} \frac{1}{n} \log \left(\sum_{\sigma^{n} x=x} \exp \left(g^{n}(x)\right)\right)
$$

on Hölder continuous functions. This map is known to be real analytic. Furthermore, the hypothesis allows us to deduce that the map $t \mapsto P\left(-t \log \left|T^{\prime}\right| \circ \pi+\right.$ $\left.q_{1} \widehat{\psi}_{1}+\ldots+q_{2 g} \widehat{\psi}_{2 g}\right)$ is strictly convex $[\mathrm{Ru}]$. We can now define the following.

Definition. Given $\underline{q}=\left(q_{1}, \ldots, q_{2 g}\right) \in \mathbb{R}^{2 g}$, we let $t=t(\underline{q}) \in \mathbb{R}$ be the (unique) solution to

$$
P\left(-t \log \left|T^{\prime}\right| \circ \pi+q_{1} \widehat{\psi}_{1}+\ldots+q_{2 g} \widehat{\psi}_{2 g}\right)=0 .
$$

Remark. Since $\log \left|T^{\prime}\right|>0$ we see that for fixed $\underline{q}$ the map $t \mapsto P\left(-t \log \left|T^{\prime}\right| \circ \pi+\right.$ $\left.q_{1} \widehat{\psi}_{1}+\ldots+q_{2 g} \widehat{\psi}_{2 g}\right)$ is a bijection on $\mathbb{R}$.

The analyticity of the map $P: C^{\gamma}\left(\Sigma_{A}^{+}\right) \rightarrow \mathbb{R}$ and the implicit function theory (using the estimate on the derivative in the next lemma) allow us to deduce that $t(\underline{q})$ has a real analytic dependence on $q$.

We also need to consider the associated Gibbs measure. Let us define a cylinder set in $\Sigma_{A}^{+}$by $\left[x_{0}, \ldots, x_{n-1}\right]=\left\{y \in \Sigma_{A}^{+}: x_{i}=y_{i}\right.$, for $\left.0 \leq i \leq n-1\right\}$. We have the following useful characterization.

Lemma 6 (cf. [Bw2]). There exists $C>0$ and a $\sigma$-invariant probabilty measure $\mu=\mu_{t, \underline{q}}$ (called $a$ Gibbs measure) such that

$$
\frac{1}{C} \leq \frac{\mu\left[x_{0}, \ldots, x_{n-1}\right]}{\left|\left(T^{n}\right)^{\prime}(\pi x)\right|^{-t(q)} e^{q_{1} \hat{\psi}_{1}^{n}(x)+\ldots+q_{2 g} \hat{\psi}_{2 g}^{n}(x)}} \leq C, \quad \forall x \in \Sigma_{A}^{+}, \quad \forall n \geq 1,
$$

where we denote $\widehat{\psi}_{i}^{n}(x)=\widehat{\psi}_{i}(x)+\widehat{\psi}_{i}(\sigma x)+\ldots+\widehat{\psi}_{i}\left(\sigma^{n-1} x\right)$, for $1 \leq i \leq 2 g$.

The next lemma shows that given $\underline{\alpha} \in \operatorname{int}(\mathcal{B})$, we can associate a Gibbs measure $\mu$.

Lemma 7. Given $\underline{\alpha} \in \operatorname{int}(\mathcal{B})$, we can find $\mu$ such that

$$
\alpha_{j}:=\frac{\int \widehat{\psi}_{j} d \mu}{\int \log \left|T^{\prime}\right| \circ \pi d \mu}, \text { for } j=1, \ldots, 2 g .
$$


Proof. Given $\underline{\alpha} \in \operatorname{int}(\mathcal{B})$, we can define

$$
\begin{aligned}
& h(\underline{\alpha}):=\sup \left\{\frac{h(m)}{\int \log \left|T^{\prime}(x)\right| d m}:\right. \\
& \left.\quad m=T \text {-invariant probability with } \frac{\int \widehat{\Psi} d m}{\int \log \left|T^{\prime}(x)\right| d m}=\underline{\alpha}\right\},
\end{aligned}
$$

where $\widehat{\Psi}=\left(\widehat{\psi}_{1}, \ldots, \widehat{\psi}_{2 g}\right)$. In particular, given any $\epsilon>0$ we can choose $m$ such that $h(m) \geq(h(\underline{\alpha})-\epsilon) \int \log \left|T^{\prime}\right| d m$ and then by the variational principle:

$$
\begin{aligned}
& P\left(-h(\underline{\alpha}) \log \left|T^{\prime}\right|+\sum_{i=1}^{2 g} q_{i}\left(\bar{\psi}_{i}-\alpha_{i} \log \left|T^{\prime}\right|\right)\right) \\
& \geq h(m)+\int\left(-h(\underline{\alpha}) \log \left|T^{\prime}\right|+\sum_{i=1}^{2 g} q_{i}\left(\bar{\psi}_{i}-\alpha_{i} \log \left|T^{\prime}\right|\right)\right) d m \\
& \geq-\epsilon \int \log \left|T^{\prime}\right| d m+\underbrace{\int\left(\sum_{i=1}^{2 g} q_{i}\left(\bar{\psi}_{i}-\alpha_{i} \log \left|T^{\prime}\right|\right)\right) d m}_{=0} .
\end{aligned}
$$

Since $\epsilon$ is arbitrary, we deduce that

$$
P\left(-h(\underline{\alpha}) \log \left|T^{\prime}\right|+\sum_{i=1}^{2 g} q_{i}\left(\bar{\psi}_{i}-\alpha_{i} \log \left|T^{\prime}\right|\right)\right) \geq 0,
$$

for all $q \in \mathbb{R}^{2 g}$. Considering (2.3) with $\underline{q} /\|q\|_{2} \in \mathbb{R}^{2 g}$ replacing $q$ we can deduce from the variational principle that there exists an invariant probabilty measure $m$, say, with

$$
\int\left(\sum_{i=1}^{2 g} q_{i}\left(\bar{\psi}_{i}-\alpha_{i} \log \left|T^{\prime}\right|\right)\right) d m \geq\|\underline{q}\|_{2} h(\underline{\alpha}) \int \log \left|T^{\prime}\right| d m \geq\|\underline{q}\|_{2}>0 .
$$

In particular, the function in (2.3) tends to infinity as $\|\underline{q}\|_{2} \rightarrow \infty$ and, by convexity of pressure, we can see that there is a unique minimum, attained at $\underline{q}=\underline{q}(\alpha)$, say. If $\mu_{q}$ is the Gibbs measure for

$$
-h(\underline{\alpha}) \log \left|T^{\prime}\right|+\sum_{i=1}^{2 g} q_{i}(\alpha)\left(\bar{\psi}_{i}-\alpha_{i} \log \left|T^{\prime}\right|\right),
$$

then for each $1 \leq i \leq 2 g$, the $i$ th partial derivative at $\underline{q}(\alpha)$ is precisely

$$
\int\left(\bar{\psi}_{i}-\alpha_{i} \log \left|T^{\prime}\right|\right) d \mu_{q}
$$

which vanishes by assumption. Thus $\int \widehat{\Psi} d \mu_{q} / \int \log \left|T^{\prime}(x)\right| d \mu_{q}=\underline{\alpha}$. Moreover,

$$
\begin{aligned}
& P\left(-h(\underline{\alpha}) \log \left|T^{\prime}\right|+\sum_{i=1}^{2 g} q_{i}\left(\bar{\psi}_{i}-\alpha_{i} \log \left|T^{\prime}\right|\right)\right) \\
& =\underbrace{h\left(\mu_{q}\right)-h(\underline{\alpha}) \int \log \left|T^{\prime}\right| d \mu_{q}}_{\leq 0}+\sum_{i=1}^{2 g} q_{i} \underbrace{\int\left(\bar{\psi}_{i}-\alpha_{i} \log \left|T^{\prime}\right|\right) d \mu_{q}}_{=0} \leq 0 .
\end{aligned}
$$


Comparing (2.3) and (2.4) we see that

$$
P\left(-\left(h(\underline{\alpha})+\sum_{i=1}^{2 g} \alpha_{i}\right) \log \left|T^{\prime}\right|+\sum_{i=1}^{2 g} q_{i} \bar{\psi}_{i}\right)=0 .
$$

In particular, the result follows with $t(\underline{q})=\left(h(\underline{\alpha})+\sum_{i=1}^{2 g} \alpha_{i}\right)$.

Finally, we have the following identities.

Lemma 8. We have that: (1) $\alpha_{j}=\partial t / \partial q_{j}$; and $(2) \mu\left(\widehat{Y}_{\underline{\alpha}}\right)=1$.

Proof. For part (1) we know by the formula for the derivative of pressure [Ru] that

$$
\left.\frac{\partial}{\partial t} P\left(-t \log \left|T^{\prime}\right| \circ \pi+q_{1} \widehat{\psi}_{1}+\cdots+q_{2 g} \widehat{\psi}_{2 g}\right)\right|_{t=t(\underline{q})}=-\int \log \left|T^{\prime}\right| \circ \pi d \mu
$$

and

$$
\left.\frac{\partial}{\partial q_{j}} P\left(-t(\underline{q}) \log \left|T^{\prime}\right| \circ \pi+q_{1} \widehat{\psi}_{1}+\cdots+q_{2 g} \widehat{\psi}_{2 g}\right)\right|_{q=q_{j}}=-\int \widehat{\psi}_{j} d \mu .
$$

The result then follows from the implicit function theorem.

Part (2) follows from the Birkhoff ergodic theorem: For a.e. $(\mu) x$ we have that

$\lim _{n \rightarrow+\infty} \frac{1}{n} \sum_{i=1}^{n} \log \left|T^{\prime} \circ \pi\left(\sigma^{i} x\right)\right|=\int \log \left|T^{\prime}\right| \circ \pi d \mu$ and $\lim _{n \rightarrow+\infty} \frac{1}{n} \sum_{i=1}^{n} \widehat{\psi}_{j}\left(\sigma^{i} x\right)=\int \widehat{\psi}_{j} d \mu$, for $j=1, \ldots, 2 g$. Using $(2.2)$ we have that $\mu\left(\widehat{Y}_{\underline{\alpha}}\right)=1$, as required.

\section{Pointwise Dimensions}

In this section we show that Lemma 4 follows from properties of pointwise dimension. This is a straightforward modification of the approach in $[\mathrm{PW}]$ for a single function. For convenience of notation, we denote $\nu=\pi^{*} \mu$ on $I$.

Definition. Given a point $\omega \in I$, we define the upper pointwise dimension and lower pointwise dimension to be

$$
\bar{d}_{\nu}(\omega)=\limsup _{r \rightarrow 0} \frac{\log \nu(B(\omega, r))}{\log r} \text { and } \underline{d}_{\nu}(\omega)=\liminf _{r \rightarrow 0} \frac{\log \nu(B(\omega, r))}{\log r},
$$

respectively.

The next lemma gives bounds on these densities in terms of quantities we defined in the previous section.

\section{Lemma 9.}

(i) For a.e. $(\nu) \omega \in Y_{\underline{\alpha}}$ we can bound $\underline{d}_{\nu}(\omega) \geq t(\underline{q})+q_{1} \alpha_{1}+\ldots+q_{2 g} \alpha_{2 g}$.

(ii) For all $\omega \in Y_{\underline{\alpha}}$ we can bound $\bar{d}_{\nu}(\omega) \leq t(\underline{q})+q_{1} \alpha_{1}+\ldots+q_{2 g} \alpha_{2 g}$.

The proof of Lemma 9 is presented in the next section. Assuming this result, we can now complete the proof of Proposition 1.

Lemma 10. For $\underline{\alpha}=\left(\alpha_{1}, \ldots, \alpha_{2 g}\right) \in \mathbb{R}^{2 g}$ and $\underline{q}=\left(q_{1}, \ldots, q_{2 g}\right) \in \mathbb{R}^{2 g}$ related as above, we can identify

$$
\operatorname{dim}_{H}\left(Y_{\underline{\alpha}}\right)=t(\underline{q})+q_{1} \alpha_{1}+\cdots+q_{2 g} \alpha_{2 g} .
$$


Proof. It is a standard result that if there exists $d$ such that $\underline{d}_{\nu}(\omega) \geq d$ for a.e. $(\nu)$ $\omega \in Y_{\alpha}$, then $\operatorname{dim}_{H}(Y) \geq d[\mathrm{Pe}$, Theorem 7.1]. Thus we deduce from Lemma 9 (i) that $\operatorname{dim}_{H}(Y) \geq t\left(q_{1}, \ldots, q_{2 g}\right)+q_{1} \alpha_{1}+\cdots+q_{2 g} \alpha_{2 g}$.

By a folklore theorem [Fa], if there exists $d$ such that $\bar{d}_{\nu}(\omega) \leq d$ for every $\omega \in Y_{\alpha}$, this implies that $\operatorname{dim}_{H}(Y) \geq d$. Taking $d=t(\underline{q})+q_{1} \alpha_{1}+\cdots+q_{2 g} \alpha_{2 g}$, by Lemma 9 (ii) we get that $\operatorname{dim}_{H}\left(Y_{\underline{\alpha}}\right) \geq t(\underline{q})+q_{1} \alpha_{1}+\cdots+q_{2 g} \alpha_{2 g}$.

The following lemma helps complete the proof of Lemma 4 (1).

Lemma 11. The map $\underline{\alpha} \mapsto \operatorname{dim}_{H}\left(Y_{\underline{\alpha}}\right), \alpha \in \operatorname{int}(\mathcal{B})$, is analytic and strictly convex.

Proof. The analyticity of $\operatorname{dim}_{H}\left(Y_{\underline{\alpha}}\right)$ follows from the analyticity of $\underline{q}(\underline{\alpha})$, which in turn follows from its characterization (in the proof of Lemma 7), the analyticity of the pressure and the implicit function theorem.

The convexity of $\underline{q} \mapsto t(\underline{q})$ is easily checked to be strictly convex by showing that the second derivative is positive definite. More precisely, by analogy with the one-dimensional case [Pe, p. 212] we can compute

$$
D^{2} t(\underline{q})\left(v_{1}, v_{2}\right)=\frac{D^{2} P\left(-\log \left|T^{\prime}\right|-\left\langle D t\left(v_{1}\right) \bar{\Psi}\right\rangle,-\log \left|T^{\prime}\right|-\left\langle D t\left(v_{2}\right) \bar{\Psi}\right\rangle\right)}{\int \log \left|T^{\prime}\right| d \mu} .
$$

The expression in (3.1) is the Legendre transform of $t(\underline{q})$ and thus, again, is strictly convex [Ar, p. 64].

By convexity we see that $\operatorname{dim}_{H}\left(Y_{\underline{\alpha}}\right) \leq 1$, with equality when $\underline{\alpha}=0$ [PW]. We can associate a Gibbs measure $m$ with $m\left(X_{\underline{\alpha}}\right)=1$. Since Gibbs measures are fully supported, we can deduce that $Y_{\underline{\alpha}}(T, S) \subset \bar{I}$ is uncountable and dense, and thus $X_{\underline{\alpha}} \subset S_{1} V$ is also dense and uncountable, as required.

\section{Proof of Lemma 9}

We give the postponed proof of Lemma 9 .

Proof of Lemma 9 (i). Fix $\epsilon>0$. For each $w \in Y_{\underline{\alpha}}$ we can choose $N(\omega)$ sufficiently large so that for $n \geq N(\omega)$ we have:

$$
\alpha_{j}-\epsilon \leq \frac{\sum_{i=0}^{n-1} \psi_{j}\left(T^{i} \omega\right)}{\sum_{i=0}^{n-1} \log \left|T^{\prime}\left(T^{i} \omega\right)\right|} \leq \alpha_{j}+\epsilon, \quad \forall 1 \leq j \leq 2 g,
$$

by Lemma 8 (2). For each $l \in \mathbb{N}$ let $Q_{l}=\{\omega: N(\omega) \leq l\}$ and observe that $Q_{l} \subset Q_{l+1}$ and $Y_{\underline{\alpha}}=\bigcup_{l=1}^{\infty} Q_{l}$ (up to a set of zero measure). Choose $l_{0}$ sufficiently large that $\mu_{q}\left(Q_{l}\right)>0$ whenever $l \geq l_{0}$.

For $l_{0}$ fixed, choose $l \geq l_{0}$. Given $0<r<1$, we can cover $\pi^{-1}\left(Q_{l}\right) \subset \Sigma_{A}^{+}$by cylinders $C^{(i)}, i=1, \ldots, N$, of length $n(i)$ based at points $x_{i} \in \pi^{-1}\left(Q_{l}\right)$, say, and length $n=n(x, r)$ such that

$$
\left|\left(T^{n(i)}\right)^{\prime}\left(\pi\left(x_{i}\right)\right)\right| \leq r<\left|\left(T^{n(i)-1}\right)^{\prime}\left(\pi\left(x_{i}\right)\right)\right| .
$$

(This called a Moran cover Pe].) Moreover, we can assume, without loss of generality, that the length of each cylinder is at least $l_{0}$. Using successively (2.1), (4.1) 
and (4.2) we can bound for any $\omega \in Q_{l}$ :

$$
\begin{aligned}
\nu\left(B(\omega, r) \cap Q_{l}\right) & \leq \sum_{i=1}^{N} \mu\left(C^{(i)}\right) \\
& \leq \sum_{i=1}^{N} C\left(\left|\left(T^{n(i)}\right)^{\prime}\left(\pi x_{i}\right)\right|^{-t(\underline{q})} e^{q_{1} \widehat{\psi}_{1}^{n(i)}\left(x_{i}\right)+\ldots+q_{2 g} \widehat{\psi}_{2 g}^{n(i)}\left(x_{i}\right)}\right) \\
& \leq \sum_{i=1}^{N} C\left(\left|\left(T^{n(i)}\right)^{\prime}\left(\pi x_{i}\right)\right|^{-t(\underline{q})-\sum_{j=1}^{2 g} q_{j}\left(\alpha_{j}-\epsilon\right)}\right) \\
& \leq C^{\prime} r^{t(\underline{q})+\sum_{j=1}^{2 g} q_{j}\left(\alpha_{j}-\epsilon\right)},
\end{aligned}
$$

for some constant $C^{\prime}>0$. By the Borel density theorem we have that for $\omega$ in a full measure (i.e., essential) set of $Q_{l}$ there exists $r_{0}(\omega)>0$ such that

$$
\nu(B(x, r)) \leq 2 \nu\left(B(x, r) \cap Q_{l}\right), \text { whenever } r<r_{0}(\omega) .
$$

In particular, comparing (4.3) and (4.4) shows that for any $l>l_{0}$ and a.e. $(\mu) \omega \in Q_{l}$ we have

$$
\underline{d}_{\nu}(\omega)=\liminf _{r \rightarrow 0} \frac{\log \nu(B(\omega, r))}{\log r} \geq t(\underline{q})+\sum_{j=1}^{2 g} q_{j}\left(\alpha_{j}-\epsilon\right) .
$$

Since $l$ and $\epsilon>0$ are arbitrary we deduce that (4.5) holds for a.e. $(\nu) \omega \in Y_{\alpha}$, as required.

Remark. Notice that the only place where we used that we have used $2 g$ limits (rather than the usual single limit as in [PW]) is in (4.1). This only influences the definition $N(\omega)$ and, thus, that of $Q_{l}$, but does not change the usual proof in any other way.

Proof of Lemma 9 (ii). Fix $r>0$. Let $\pi(x)=\omega \in I$ and choose $n=n(x, r)$ precisely so that

$$
\left|\left(T^{n}\right)^{\prime}(\omega)\right|^{-1} \leq r<\left|\left(T^{n-1}\right)^{\prime}(\omega)\right|^{-1} .
$$

In particular, $\pi\left(\left[x_{0}, \ldots, x_{2 g}\right]\right) \subset B(\omega, D r)$, for some $D>0$. Thus for all $\omega \in Y_{\underline{\alpha}}$ we have by (2.1), (4.1) and (4.6),

$$
\begin{aligned}
\nu(B(\omega, D r)) & \geq \mu\left(\left[x_{0}, \ldots, x_{m}\right]\right) \\
& \geq \frac{1}{C}\left|\left(T^{n}\right)^{\prime}(\pi x)\right|^{-t(\underline{q})} e^{q_{1} \widehat{\psi}_{1}^{n}(x)+\ldots+q_{2 g} \widehat{\psi}_{2 g}^{n}(x)} \\
& \geq \frac{1}{C}\left|\left(T^{n}\right)^{\prime}(\pi x)\right|^{-t(\underline{q})-\sum_{j=1}^{2 g} q_{j}\left(\alpha_{j}+\epsilon\right)} \\
& \geq \frac{1}{C^{\prime \prime}} r^{t(\underline{q})+\sum_{j=1}^{2 g} q_{j}\left(\alpha_{j}+\epsilon\right)},
\end{aligned}
$$

for some constant $C^{\prime \prime}>0$. Thus

$$
\bar{d}_{\nu}(x)=\limsup _{r \rightarrow 0} \frac{\log \nu_{q}(B(x, r))}{\log r} \leq t(\underline{q})+\sum_{j=1}^{2 g} q_{j}\left(\alpha_{j}+\epsilon\right) .
$$

Since $\epsilon>0$ was arbitrary, we deduce that $\bar{d}_{\nu}(x) \leq t+\sum_{j=1}^{2 g} q_{j} \alpha_{j}$. 
Remark. A similar problem relates to the set $\mathcal{P}=\left\{\int z d \mu: \mu=T\right.$-invariant $\} \subset \mathbb{C}$ of complex numbers which occur as the first Fourier coefficient of invariant measures for a $C^{2}$ expanding map $T: K \rightarrow K$ on the unit circle $K=\{z \in \mathbb{C}:|z|=1\}$. The set

$$
\mathcal{P}=\left\{\int z d \mu(z) \in \mathbb{C}: \mu=T \text {-invariant probability }\right\}
$$

has been extensively studied by Jenkinson JJe1, Bousch Bo and others, particularly in the case $T(z)=z^{2}$. For a typical point $z$ on the unit circle the Birkhoff averages $\frac{1}{N} \sum_{n=1}^{N} T^{n}(z)$ converge to zero. We can denote $K_{\omega}=\{x \in$ $\left.X: \lim _{N \rightarrow+\infty} \frac{1}{N} \sum_{n=1}^{N} T^{n}(x)=\omega\right\}$, which has zero Liouville measure for $\omega \neq 0$. The analysis in this note shows that for $\underline{\alpha} \in \operatorname{int}(\mathcal{P})$ we have that:

(1) The Hausdorff $\operatorname{dimension}_{\operatorname{dim}_{H}}\left(X_{\omega}\right)$ satisfies $\operatorname{dim}_{H}\left(X_{\omega}\right) \leq 1$, with equality if and only if $\omega=0$;

(2) The Hausdorff dimension $\operatorname{dim}_{H}\left(X_{\underline{\alpha}}\right)$ depends real analytically on $\underline{\alpha} \in$ $\operatorname{int}(\mathcal{P})$

(3) $X_{\omega} \subset I$ is dense, uncountable and carries a Gibbs measure.

For the particular case of the doubling map $T(z)=z^{2}$, this problem has been studied by Fan and Schmeling [FS] and the Birkhoff averages take the simple form $\frac{1}{N} \sum_{n=1}^{N} z^{2^{k}}$.

\section{REFERENCES}

[Ar] V. Arnold, Mathematical Methods of Classical Mechanics, Graduate Texts in Mathematics, 60, Springer-Verlag, Berlin, 1978. MR 57:14033b

[Ba] V. Bangert, Minimal measures and minimizing closed normal one-currents, Geom. Funct. Anal. 9 (1999), 413-427. MR 2000m:49058

[BSS] L. Barreira, B. Saussol, and J. Schmeling, Higher-dimensional multifractal analysis, J. Math. Pures Appl. 81 (2002), 67-91.

[Bo] T. Bousch, Le poisson n'a pas d'arêtes, Ann. Inst. Henri Poincaré Probab. Statist. 36 (2000), 489-508. MR 2001i:37005

[Bw1] R. Bowen, Symbolic dynamics for hyperbolic flows, Amer. J. Math. 95 (1973), 429-460. MR 49:4041

[Bw2] R. Bowen, Equilibrium states and the ergodic theory of Anosov diffeomorphisms, Lecture Notes in Mathematics, Vol. 470, Springer-Verlag, Berlin, 1975. MR 56:1364

[BI] D. Burago and S. Ivanov, Riemannian tori without conjugate points are flat, Geom. Funct. Anal. 4 (1994), 259-269. MR 95h:53049

[Fa] K. Falconer, Fractal geometry, John Wiley, Chichester, 1990. MR 92j:28008

[FS] A. H. Fan and J. Schmeling, On fast Birkhoff averaging, Math. Proc. Cambridge Philos. Soc., to appear.

[Fr] J. Franks, Knots, links and symbolic dynamics., Annals of Math. 113 (1981), 529-552. MR 83h:58074

[Je1] O. Jenkinson, Frequency locking on the boundary of the barycentre set, Experiment. Math. 9 (2000), 309-317. MR 2001g:37050

[Je2] O. Jenkinson, Rotation, Entropy, and Equilibrium States, Trans. Amer. Math. Soc. 353 (2001), 3713-3739. MR 2002e:37004

[Ma1] D. Massart, Ph.D. Thesis, (Lyon).

[Ma2] D. Massart, Stable norms of surfaces: local structure of the unit ball of rational directions, Geom. Funct. Anal. 7 (1997), 996-1010. MR 99b:53061

[MR] G. McShane and I. Rivin, Simple curves on hyperbolic tori, C. R. Acad. Sci. Paris Sér. I Math. 320 (1995), 1523-1528. MR 96g:57018

[Pe] Y. Pesin, Dimension theory in dynamical systems., Chicago Lectures in Mathematics, University of Chicago Press, Chicago, 1997. MR 99b:58003

[PS] Y. Pesin and V. Sadovskaya, Multifractal analysis of conformal Axiom A flows, Comm. Math. Phys. 216 (2001), 277-312. MR 2002g:37035 
[PW] Y. Pesin and H. Weiss, The multifractal analysis of Gibbs measures: motivation, mathematical foundation, and examples, Chaos 7 (1997), 89-106. MR 98e:58130

[Ra] M. Ratner, Markov partitions for Anosov flows on $n$-dimensional manifolds, Israel J. Math. 15 (1973), 92-114. MR 49:4042

[Sc] S. Schwartzman, Asymptotic cycles, Ann. of Math. 66 (1957), 270-284. MR 19:568i

[Ru] D. Ruelle, Thermodynamic Formalism, Addison-Wesley Publ. Co., Reading, MA, 1978. MR 80g:82017

[Su] D. Sullivan, Cycles for the dynamical study of foliated manifolds and complex manifolds, Invent. Math. 36 (1976), 225-255. MR 55:6440

Department of Mathematics, University of Manchester, Oxford Road, Manchester, M13 9PL, ENGLAND 